\title{
A Perspective on Modal Sequent Logic
}

\author{
By \\ Stephen BlameY* and Lloyd Humberstone**
}

\section{§0. Prefatory Remarks}

This paper was drafted in 1981-2 when the first author was lecturing on modal logic for the Philosophy Subfaculty at Oxford University and the second author was visiting Oxford on study leave; it was revised the following year. ${ }^{1)}$ Both of us had been graduate students of D. S. Scott at Oxford in the 1970's and were impressed by his emphasis on the desirability of isolating the structural properties of a (logical) consequence relation - such as are encoded in the principles $(\boldsymbol{R}),(\boldsymbol{M})$, and $(\boldsymbol{T})$ of [11], [12] - from principles relating to specific connectives. Extending this idea to the case of the modal operators, we found that distinctions between several well-known systems of (normal) modal logic could be reflected at the purely structural level, if an appropriate notion of sequent was adopted. Actually, we work with one notion of sequent in $\$ \S 1-4$ and consider a somewhat more refined version in $\$ 5$. On later finding that sequents of the latter type had already been used by M. Sato, who, in $\$ 3.4$ of [10], credits the idea to $O$. Sonobe, we had some misgivings about publishing the material at full length. That anticipation notwithstanding, however, it appears to us still worth proceeding with a somewhat abridged version of the paper, both so as to highlight the original motivation and also because our treatment and Sato's differ on many points of detail. ${ }^{2)}$ We should mention that K. Došen, in [4], also advocates a variation on the traditional idea of what a sequent should look like for the case of modal logic. Though the framework he sets up is quite different from our own, he is in part motivated by similar consideratons (e.g., the concern with 'unique characterization' - see $\S 4$ below). Some aspects of our own way of proceeding may be seen (again, in retrospect) as steps in the execution of Belnap's 'Display Logic' programme (see [2]), in that the rules ( $\square$ ) of $\S 4$ serve

Communicated by S. Takasu, June 4, 1990.

1991 Mathematics Subject Classification: 03B45.

* Programming Research Group, Oxford Computing Laboratory, 8-11, Keble Road, Oxford. England.

** Department of Philosophy, Monash University, Wellington Road, Clayton Victoria 3168, Australia. 
to 'display' the formula in the scope of an occurrence of ' $\square$ ' standing extricated from that scope. ${ }^{3)}$

\section{\$1. Introduction}

The term 'sequent logic', as it figures in our title, is intended connote any development of a system of formal logic which proceeds by the application of rules which transform sequents into sequents. This includes the natural deduction approach, with rules recast so as to pass from sequents to sequents (thereby making the manipulation of assumption dependencies explicit), as well as sequentcalculus presentations in the style of Gentzen, in which rules governing connectives introduce those connectives on the left or the right of the sequents, as well as approaches which fall under neither of these headings: our own treatment falls into this last category. Using the symbol ' $>$ ' to separate what is on the left of a sequent from what is on the right, sequents of a more or less traditional form appear as objects $\Gamma>\Delta$, where $\Gamma$ and $\Delta$ are (possibly empty) finite sets of formulae of the language under consideration. A heuristic, semantically inspired, reading of such sequents is: if all formulae in $\Gamma$ are true then at least one formula in $\Delta$ is true. For the sake of separating out structural elements, as mentioned in $\S 0$, we work (until §5) with three-place sequents instead, writing $\Gamma \succ_{\Sigma} \Delta$, where now $\Sigma$ is, like $\Gamma$ and $\Delta$, a finite set of formulae. We may think of $\Gamma$ as representing the ordinary premisses of an argument to the (disjunctive) conclusion represented by $\Delta$, and of $\Sigma$ as representing the 'modal premisses' of this argument. A heuristic reading would be: if all formulae in $\Gamma$ are true, and all formulae in $\Sigma$ are necessary, then at least one formula in $\Delta$ is true. In particular, the distinctively modal rule of 'Necessitation' can be recorded by the sequentschema $\succ_{\varphi} \square \varphi$ (which appears as $(\square \downarrow)_{0}$ in $\S 4$ ); the attempt to record its effect by $\varphi>\square \varphi$ would of course be a mistake, given the present understanding of ' $>$ '. (In what follows, we do not consider formula-to-formula rules but only sequent-to-sequent rules, and to avoid confusion with the usage of this paragraph we will usually refer to the premisses and conclusions of their applications as premiss-sequents and conclusion-sequents.)

To make the above ideas more precise, we adapt the Kripke semantics for modal logic to our framework. Though we will not need to refer to specific connectives until $\S 4$, let us suppose that the formulae of our languages - call it $L$-are constructed from a stock of atomic formulae $p_{1}, \ldots p_{n}, \ldots$ ('propositional variables') by means of two singulary connectives $\neg$ and $\square$ and one binary connective $\wedge$. We are thinking of $\neg$ and $\wedge$ as (boolean) negation and conjunction, with $\square$ for 'it is necessary that' (though there is no intention to rule out alternative non-alethic readings).

A frame is a pair $\langle W, R\rangle$ in which $W \neq \varnothing$ and $R \subseteq W \times W$; a structure is a triple $\langle W, R, V\rangle$ in which $\langle W, R\rangle$ is a frame and $V: L \times W \rightarrow\{T, F\} . R$ and $V$ 
are referred to respectively as the accessibility relation and the valuation of the structure. A model is a structure $\langle W, R, V\rangle$ with $V$ satisfying, for all $x \in W$, all $\varphi, \psi \in L:$

$$
\begin{aligned}
& \text { [ᄀ] } V(\neg \varphi, x)=T \Leftrightarrow V(\varphi, x)=F \\
& \text { [^] } V(\varphi \wedge \psi, x)=T \Leftrightarrow V(\varphi, x)=V(\psi, x)=T \\
& \text { [ㅁ] } V(\square \varphi, x)=T \Leftrightarrow \forall y \in W . x R y \Rightarrow V(\varphi, y)=T
\end{aligned}
$$

Where $\langle W, R, V\rangle$ is any structure (not necessarily a model) and $\Gamma \succ_{\Sigma} \Delta$ is any sequent, we say $\Gamma \succ_{\Sigma} \Delta$ holds at the point $x \in W$ just in case:

If for all $\varphi \in \Gamma, V(\varphi, x)=T$ and for all $\sigma \in \Sigma, V(\sigma, y)=T$

for every $y$ such that $x R y$, then $V(\psi, x)=T$ for some $\psi \in \Delta$.

A sequent will be said to hold in a structure if it holds at every point in the structure. A sequent fails at a point/in a structure if it does not hold at that point/in that structure. Below, we shall be introducing various systems of modal sequent logic by means of certain closure conditions. These conditions can be expressed as (sequent-to-sequent) rules, so each such characterization of a class of systems can be regarded as a proof-theoretic presentation of the smallest system in the class. However, our official understanding of 'system' will be simply as a collection of sequents (satisfying whatever closure conditions are specified.) When every sequent in a system $S$ holds in a structure, we say that the given structure is a structure for $S$, and when the structure is also a model, that it is a model for $S$. Apart from an occasional aside, we will not be concerned with frames and so do not define an analogous notion 'frame for $S$ '; further, until $\S 4$, we will be concerned with arbitrary structures without specific attention to those which qualify as models. If $\mathbb{C}$ is a class of structures, a system $S$ is sound w.r.t. $\mathbb{C}$ if each sequent in $S$ holds in every structure in $C$, complete w.r.t. $C$ if each sequent holding in every structure in $C$ belongs to $S$, and determined by $C$ when both sound and complete w.r.t. $C$. Since the sequent $\varnothing>_{\varnothing} \varnothing$ holds in no structure, the 'inconsistent' system in which this sequent (along with all others) is provable is determined by the empty class of structures.

\section{§2. Some Structural Rules}

We begin by stating four fundamental structural rules, ${ }^{4}$ the first three of which - reflexivity, monotonicity and transitivity principles - are adapted from Scott (see $§ 0)$ :

(R) $\varphi>\varphi$

(M)

$\frac{\Gamma \succ_{\Sigma} \Delta}{\Gamma, \Gamma^{\prime} \succ_{\Sigma, \Sigma^{\prime}} \Delta, \Delta^{\prime}}$

$(T)$

$\frac{\Gamma, \varphi \succ_{\Sigma} \Delta \quad \Gamma \succ_{\Sigma} \varphi, \Delta}{\Gamma \succ_{{ }_{\Sigma}} \Delta}$


$(\boldsymbol{M})$ and $(\boldsymbol{T})$ are also called 'Thinning' and 'Cut', and some of our rules will be labelled with appropriate variations on these terms, beginning with the fourth of the basic structural rules, the only one which is distinctively modal:

(Undercut)

$$
\frac{\Sigma \succ \quad \Gamma \succ_{\Sigma^{\prime}, \sigma} \Delta}{\Gamma \succ_{\Sigma, \Sigma^{\prime}} \Delta}
$$

In the statement of this last rule, we write ' $\Sigma>\sigma$ ' for ' $\Sigma \succ_{\varnothing} \sigma$ '; a similar ellipsis will occur elsewhere (and, as usual, notations such as ' $\Sigma$, $\sigma$ ' stand for $\Sigma \cup\{\sigma\}$, etc.). ${ }^{5)}$ Any collection of sequents closed under these rules will be called a $\mathbb{K}^{\mathbf{3}}$ system, the superscripted ' 3 ' being intended as a reminder of the three-place sequents with which we are working here. For later reference, we observe here, and invite the reader to verify that the rules $(\mathbb{R}),(M),(T)$ and (Undercut) all preserve the property of holding in an arbitrarily selected structure. ${ }^{6}$ )

We shall prove a schematic completeness result for the above rules as Theorem 1 below. Some definitions and lemmas pave the way. In the context of discussing a system $S$ (all systems considered being $\mathbb{K}^{3}$-systems) we write ' $\Gamma \vdash_{\Sigma} \Delta$ ' to mean that there are finite subsets $\Gamma_{0}, \Sigma_{0}, \Delta_{0}$ of (the possibly infinite sets) $\Gamma$, $\Sigma, \Delta$, respectively, for which the sequent $\Gamma_{0} \succ_{\Sigma_{0}} \Delta_{0}$ belongs to $S$; an oblique stroke through the ' $F$ ' serves to negate such a claim. A triple $\langle\Gamma, \Sigma, \Delta\rangle$ is $S$ consistent iff $\Gamma \vdash_{\Sigma} \Delta$, and maximal $S$-consistent when $S$-consistent and such that any $S$-consistent $\left\langle\Gamma^{\prime}, \Sigma^{\prime}, \Delta^{\prime}\right\rangle$ with $\Gamma^{\prime} \supseteq \Gamma, \Sigma^{\prime} \supseteq \Sigma, \Delta^{\prime} \supseteq \Delta$, is actually identical with $\langle\Gamma, \Sigma, \Delta\rangle$. Finally, when we use the notation $\cap\{\Gamma \mid \ldots \Gamma \ldots\}$ for the intersection of a collection of sets of formulae, by convention we understand $\cap \emptyset$ to be the set $L$ of all formulae. The lemmas which follow pertain to any $\mathbb{K}^{3}$ system $S$, with consistency understood as $S$-consistency, etc.

Lemma $\mathbb{A}$. For any consistent triple $\langle\Gamma, \Sigma, \Delta\rangle$ there is a maximal consistent triple $\left\langle\Gamma^{\prime}, \Sigma^{\prime}, \Delta^{\prime}\right\rangle$ with $\Gamma^{\prime} \supseteq \Gamma, \Sigma^{\prime} \supseteq \Sigma, \Delta^{\prime} \supseteq \Delta$.

Proof. By a Lindenbaum construction.

Lempma $\mathbb{B}_{0}$, For any maximal consistent $\langle\Gamma, \Sigma, \Delta\rangle$ $\Sigma=\cap\left\{\Gamma^{\prime} \mid\right.$ for some $\Sigma^{\prime}, \Delta^{\prime},\left\langle\Gamma^{\prime}, \Sigma^{\prime}, \Delta^{\prime}\right\rangle$ is maximal consistent and $\left.\Sigma \subseteq \Gamma^{\prime}\right\}$

Proof. Clearly $\Sigma$ is included in the intersection in question. For the converse, suppose $\sigma \notin \Sigma$. It follows that $\Gamma \vdash_{\Sigma, \sigma} \Delta$. Hence $\Sigma \nvdash \sigma$. (Otherwsie, by (Undercut), $\Gamma \vdash_{\Sigma} \Delta$.) Therefore, by Lemma A, there is a maximal consistent $\left\langle\Gamma^{\prime}\right.$, $\left.\Sigma^{\prime}, \Delta^{\prime}\right\rangle$ such that $\Sigma \subseteq \Gamma^{\prime}$ and $\sigma \notin \Gamma^{\prime}$ (because $\sigma \in \Delta^{\prime}$ : closure of $S$ under $(\mathbb{R})$ rules out $\sigma^{\prime}$ s belonging to both these sets for $S$-consistent $\left.\left\langle\Gamma^{\prime}, \Sigma^{\prime}, \Delta^{\prime}\right\rangle\right)$.

We shall show that any $\mathbb{K}^{3}$-system $S$ is determined by the class of all its structures, i.e., by the class of all structures for $S$ (in the sense of $\S 1$ ). Since no structure is a structure for the inconsistent system, and this system, as noted at 
the end of $\S 1$, is determined by the empty class of structures, we have the result for that case and pass to the case of consistent $S$. For these $S$, following the Scott-Makinson adaptation of Henkin completeness proofs (see, e.g., [13]), the method of proof involves constructing a canonical structure whose elements are certain $S$-consistent triples. If $S$ were inconsistent, there would be no such triples, whereas in the definition of a structure $\langle W, R, V\rangle$, we have required that $W$ be a non-empty set.

More precisely, then, the canonical structure for a consistent $\mathbf{K}^{\mathbf{3}}$-system $S$ is defined to be the triple $\left\langle W_{S}, R_{S}, V_{S}\right\rangle$ in which:

$$
\begin{aligned}
& W_{S}: W_{S} \text { is the set of all maximal } S \text {-consistent triples. } \\
& R_{S}:\langle\Gamma, \Sigma, \Delta\rangle R_{S}\left\langle\Gamma^{\prime}, \Sigma^{\prime}, \Delta^{\prime}\right\rangle \text { iff } \Sigma \subseteq \Gamma^{\prime} . \\
& V_{S}: \text { For any formula } \varphi, V_{S}(\varphi,\langle\Gamma, \Sigma, \Delta\rangle)=T \text { if } \varphi \in \Gamma \\
& \qquad V_{S}(\varphi,\langle\Gamma, \Sigma, \Delta\rangle)=T \text { if } \varphi \in \Delta .
\end{aligned}
$$

A propos of $R_{S}$, we remark that in the usual two-place sequent setting for normal modal logics (as in [8], with an analogous point for the even more common 'formula' based treatments) $R_{S}$ would be defined to relate $\langle\Gamma, \Delta\rangle$ to $\left\langle\Gamma^{\prime}, \Delta^{\prime}\right\rangle$ when for all $\varphi$ such that $\square \varphi \in \Gamma, \varphi \in \Gamma^{\prime}$. Similarly, we have introduced the canonical accessibility relation to relate one element of the canonical structure to another whenever whatever is necessary according to the former is true according to the latter, but since we have introduced a special position (the ' $\Sigma$ '-position) to record the status of a formula as necessary, no specifically modal connectives need to intrude to obtain this effect. And à propos of $V_{S}$, notice that, assuming $S$ to be a $\mathbb{K}^{3}$-system, closure under $(\mathbb{R})$ and $(\mathbb{M})$ guarantees that at most one of the two conditions in the definition of $V_{S}$ can be met, while closure under $(\mathbb{T})$ guarantees that at least one is met. It follows that $\left\langle W_{S}, R_{S}\right.$, $\left.V_{S}\right\rangle$ is a structure, in our sense; that it is also a structure for $S$ will follow from

Lemmma $\mathbb{C}$. A sequent $\Gamma_{0} \succ_{\Sigma_{0}} \Delta_{0}$ fails at $\langle\Gamma, \Sigma, \Delta\rangle$ in $\left\langle W_{S}, R_{S}, V_{S}\right\rangle$ iff $\Gamma_{0} \subseteq \Gamma, \Sigma_{0} \subseteq \Sigma$, and $\Delta_{0} \subseteq \Delta$.

Proof. 'If': immediate, from the definitions of $R_{S}$ and $V_{S}$. 'Only if': Suppose it is not the case that $\Gamma_{0} \subseteq \Gamma$. Then for some $\varphi \in \Gamma_{0}, \varphi \notin \Gamma$, and so $V_{S}(\varphi,\langle\Gamma, \Sigma$, $\Delta\rangle)=F$. Since a necessary condition for $\Gamma_{0} \succ_{\Sigma_{0}} \Delta_{0}$ to fail at $\langle\Gamma, \Sigma, \Delta\rangle$ is that $V(\psi,\langle\Gamma, \Sigma, \Delta\rangle)=T$ for all $\psi \in \Gamma_{0}$, it follows that $\Gamma_{0} \succ_{\Sigma_{0}} \Delta_{0}$ holds at $\langle\Gamma, \Sigma, \Delta\rangle$. Similar reasoning applies to the supposition that not $\Delta_{0} \subseteq \Delta$. So finally, suppose that not $\Sigma_{0} \subseteq \Sigma$. Then for some $\sigma \in \Sigma_{0}, \sigma \notin \Sigma$. So by Lemma $\mathbb{B}$ there is some $\left\langle\Gamma^{\prime}, \Sigma^{\prime}, \Delta^{\prime}\right\rangle \in W_{S}$ such that $\Sigma \subseteq \Gamma^{\prime}$ and $\sigma \notin \Gamma^{\prime}$. Hence $\langle\Gamma, \Sigma, \Delta\rangle R_{S}\left\langle\Gamma^{\prime}, \Sigma^{\prime}\right.$, $\left.\Delta^{\prime}\right\rangle$; but $V_{S}\left(\sigma,\left\langle\Gamma^{\prime}, \Sigma^{\prime}, \Delta^{\prime}\right\rangle\right)=F$. Thus again a necessary condition for the sequent $\Gamma_{0} \succ_{\Sigma_{0}} \Delta_{0}$ to fail at $\langle\Gamma, \Sigma, \Delta\rangle$ is lacking. 
Theorem 1. Any $\mathbf{K}^{3}$-system $S$ is determined by the class of all its structures.

Proof. That $S$ is sound w.r.t the class of all its structures is trivial, since any structure in which a sequent in $S$ fails is, by definition, not a structure for $S$.

That $S$ is complete w.r.t. the class of all its structures has already been remarked for the case of inconsistent $S$. For $S$ consistent, the result will follow if for any sequent not belonging to $S$ we can exhibit a structure for $S$ in which that sequent does not hold. But the canonical structure $\left\langle W_{S}, R_{S}, V_{S}\right\rangle$ will be seen to suffice in this capacity for all sequents not in $S$ at once, provided we check that (1) it is a structure for $S$, and (2) only sequents in $S$ hold in this structure.

As to (1): If the sequent $\Gamma_{0} \succ_{\Sigma_{0}} \Delta_{0}$ belongs to $S$ then $\Gamma \vdash_{\Sigma} \Delta$ for any $\Gamma \supseteq \Gamma_{0}$, $\Delta \supseteq \Delta_{0}, \Sigma \supseteq \Sigma_{0}$, and so no such triple $\langle\Gamma, \Sigma, \Delta\rangle$ is $S$-consistent: thus none are to be found amongst the elements of $W_{S}$. But the 'only if' part of Lemma C assures us that it is only at such triples that $\Gamma_{0} \succ_{\Sigma_{0}} \Delta_{0}$ can fail.

As to (2): If the sequent $\Gamma_{0} \succ_{\Sigma_{0}} \Delta_{0}$ does not belong to $S$ then the $S$-consistent triple $\left\langle\Gamma_{0}, \Sigma_{0}, \Delta_{0}\right\rangle$ extends (by Lemma A) to an element of $W_{S}$, at which, by the 'if' half of Lemma $\mathrm{C}$, the sequent fails.

Remark. As the above proof reveals, a stronger statement than Theorem 1 has been established: Any $\mathbb{K}^{3}$-system $S$ is determined by its canonical structure.

We turn now to some further structural rules, with a corresponding collection of completeness results gathered together as Theorem 2. Any $\mathbf{K}^{3}$-system containing all sequents of the form:

$$
\succ_{\sigma} \sigma
$$

will be called a $\mathbb{K}^{3}$-system. Equivalently, one could define a $\mathbb{K}^{\mathbf{T}}{ }^{3}$-system to be any $\mathbb{K}^{3}$-system closed under the rule:

(Downward shift)

$$
\frac{\Gamma, \sigma \succ_{\Sigma} \Delta}{\Gamma \succ_{\Sigma, \sigma} \Delta}
$$

Applying this rule repeatedly, we see that closure under it implies (and is implied by: take $\Sigma^{\prime}=\{\sigma\}$ ) the rule

$$
\frac{\Gamma, \Sigma^{\prime}>_{\Sigma} \Delta}{\Gamma \succ_{\Sigma, \Sigma^{\prime}} \Delta}
$$

whose special case, with $\Gamma=\Delta=\Sigma=\varnothing$ we call

$$
\frac{\Sigma^{\prime}>}{>_{\Sigma^{\prime}}}
$$

Any $\mathbf{K}^{3}$-system closed under this rule we call a $\mathbf{K D}^{\mathbf{3}}$-system. 
The classes of $\mathbf{K}^{3}$-system, $\mathbf{K T}^{\mathbf{3}}$-systems, and $\mathbf{K D}^{3}$-systems are so named, because the smallest such systems closed under the operational rules of $\$ 4$ below are our three-place sequent logics corresponding to the well-known normal modal systems $\mathbf{K}, \mathbf{K T}$, and $\mathbf{K D} .^{7)} \mathbf{K}$ is the least such system, and the inconsistent system the strongest. As is also well known, there are two Post-complete normal modal logics, sometimes called the 'Trivial System' and the 'Verum' (or 'Absurd') system, dual atoms in the lattice of all such logics. They are conveniently broached here; our label in the former case is derived from Chellas [3]. Let a $\mathbb{K T}^{3}{ }^{3}$-system be any $\mathbf{K T}^{3}$-system closed under the following inverted form of (Downward Shift):

(Upward Shift)

$$
\frac{\Gamma \succ_{\Sigma, \sigma} \Delta}{\Gamma, \sigma \succ_{\Sigma} \Delta}
$$

We can of course study the effects of (Upward Shift) in isolation from (Downward Shift) (or $\left(\mathbf{T}^{\mathbf{3}}\right)$ ), and, again following the notation of [3], let us call any $\mathbf{K}^{\mathbf{3}}$ system closed under (Upward Shift) a $\mathbf{K T}_{\mathbf{c}^{3}}{ }^{3}$-system. A $\mathbf{K V e r}{ }^{3}$-system, finally, will be any $\mathbf{K}^{\mathbf{3}}$-system closed under the rule:

$\left(\operatorname{Ver}^{3}\right)$

$$
\frac{\Gamma \succ_{\Sigma} \Delta}{\Gamma \succ \Delta}
$$

The analogue of Theorem 1, for these more restricted classes of systems, is the following.

Theorem 2. If $S$ is (1) any $\mathbf{K T}^{\mathbf{3}}$-system, (2) any $\mathbf{K D}^{\mathbf{3}}$-system, (3) any $\mathbf{K T}_{\mathbf{c}^{-}}^{\mathbf{3}}$ system, (4) any $\mathbf{K V e r}^{3}$-system, then $S$ is determined by the class of all its structures $\langle W, R, V\rangle$ in which, for (1): $R$ is reflexive; for (2): $R$ is serial; for (3): $x R y$ only if $\{\varphi \mid V(\varphi, x)=T\} \subseteq\{\varphi / V(\varphi, y)=T\}$; and for (4): $R$ is empty.

Proof. For the reasons given in the proof of Theorem 1, it will suffice to show in each case that the canonical structure for $S$ meets the cited condition. In fact, we give the proof only for case (1).

Suppose $S$ is a $\mathbf{K T}^{\mathbf{3}}$-system and some $\langle\Gamma, \Sigma, \Delta\rangle \in W_{S}$ does not bear the relation $R_{S}$ to itself. Thus for some $\sigma \in \Sigma, \sigma \notin \Gamma$. By maximality, then, $\Gamma, \sigma \vdash_{\Sigma} \Delta$, and so by (Downward Shift) $\Gamma \vdash_{\Sigma, \sigma} \Delta$ : but this is impossible since ex hypothesi $\sigma \in \Sigma$ and $\Gamma H_{\Sigma} \Delta$.

Part (3) of the Theorem is somewhat surprising, in that one might have expected the following condition on structures for this case: $x R y$ only if $x=y$. Then this, like the other conditions, would have been (in effect) a condition on the frames of the structures and would accord with familiar experience in modal logic (where $\mathbf{K T}_{\boldsymbol{c}}$ is determined by the class of all frames meeting the condition just cited). It turns out that if $S$ is a $\mathbf{K T}_{c^{3}}$-system closed under the classical rules governing negation (see $\$ 4$ ) then this stronger conclusion can be obtained, since 
in this case for $\langle\Gamma, \Sigma, \Delta\rangle,\left\langle\Gamma^{\prime}, \Sigma^{\prime}, \Delta^{\prime}\right\rangle \in W_{S}$, if $\Gamma \subseteq \Gamma^{\prime}$ then $\Gamma=\Gamma^{\prime}$ (and so $\Delta=$ $\Delta^{\prime}$ ), from which it would follow if $S$ were also closed under the rules for $\square$ in $\S 4$ that $\Sigma=\Sigma^{\prime}$ (since $\Sigma$ is then $\{\varphi \mid \square \varphi \in \Gamma\}{ }^{8}$ ) Indeed even without the $\square$-rules, we note that for $S$ a $\mathbf{K T}^{\mathbf{y}^{3}}$-system and $\langle\Gamma, \Sigma, \Delta\rangle \in W_{S}$ we have $\Gamma=\Sigma$ and so $\Gamma=\Gamma^{\prime}$ implies $\Sigma=\Sigma^{\prime}$. (The proof of (1) gives that for $S$ a $\mathbb{K}^{T^{3}}$-system, $\Sigma \subseteq \Gamma$ by (Downward Shift), and a similar argument invoking (Upward Shift) gives the converse inclusion.)

We have already noted that the rules $(\mathbb{R}),(\boldsymbol{M}),(\mathbb{T})$ and (Undercut) preserve, for an arbitrary structure, the property of holding in that structure. Having since introduced further rules for consideration, we should at this point register a similar observation in their case for future $(\$ 4)$ reference: namely that the rules used as closure conditions to characterize the classes of systems figuring in Theorem 2 preserve the property of holding in any given structure meeting the corresponding condition. By way of illustration, we check this explicitly for the $\mathrm{KT}^{3}$-systems, which could be done by showing that the rule (Downward shift) always leads from a sequent holding in a reflexive structure to a sequent holding in that structure, or, more simply, by showing that every instance of the schema $\left(\mathbb{T}^{3}\right)$ holds in every reflexive structure. This is obvious enough: if $>_{\sigma} \sigma$ fails in a structure $\langle W, R, V\rangle$ then there is $x \in W$ with $V(\sigma, y)=T$ for all $y$ such that $x R y$, but $V(\sigma, x)=F$; and clearly this cannot happen if $x$ is itself a $y$ such that $x R y$. Such results play a part in proving the soundness w.r.t. a class of reflexive structures of particular $\mathbb{K}^{3}$-systems in $\S 4$. Of course, a given such system will not be expected to be sound w.r.t. the class of all reflexive structures, since it will typically contain sequents over the above those guaranteed by the rules (the $\mathbb{K}^{3}$ rules and $\left(\mathbb{T}^{3}\right)$ ) used to delimit this class of systems - sequents which may fail in some reflexive structures. (And similarly, mutatis mutandis, for the other classes of systems and corresponding conditions on structures.)

\section{§3. Tramsitivity}

In this section, we approach from our point of view the familiar modal logics $\mathbb{K} \$$ and $\mathbf{S} 4$. The strategy is as in $\S 2$ : we use purely structural rules to characterize classes of systems, for which we obtain an 'abstract' completeness result along the lines of Theorems 1 and 2. But the tactics are somewhat different. As will become apparent, a slight variation on the method outlined above for the construction of canonical structures turns out to be called for.

To introduce the topic, we coin a new piece of terminology. Let us say that, understanding ' $\vdash$ ' in relation to some system $S$, when $\vdash_{\sigma} \varphi$, the formula $\sigma$ yields the formula $\varphi$ in $S$. Then the condition $\left(\mathbb{T}^{3}\right)$ on $\mathbb{K}^{3}$-systems says that each formula yields itself in these systems. Accordingly, there is an association, for such systems not only with the reflexivity of the accessibility relation in the structures with which we may treat them semantically, but also with the reflexivity 
of the syntactically defined 'yields' relation. While we do not here enter into a general investigation of such parallels, we note that the above situation also obtains in respect of transitivity, encountered in the usual model-theoretic treatment of extensions of $\mathbf{K} \mathbf{4}$ (cf. Theorem 3 below) and also here in the form of a condition on the 'yields' relation: the condition that for any $\sigma, \varphi, \psi$ if $\vdash_{\sigma} \varphi$ and $\vdash_{\varphi} \psi$ then $\vdash_{\sigma} \psi$.

Actually, rather than directly translating this condition into 'rule' form, we prefer a slightly more decked-out formulation:

(Cross-cut)

$$
\frac{\succ_{\Sigma} \sigma \quad \Gamma \succ_{\Sigma^{\prime},{ }_{\sigma}} \Delta}{\Gamma \succ_{\Sigma, \Sigma^{\prime}} \Delta}
$$

The rule is so named because we 'cut' the formula $\sigma$ here across categories: it appears as an ordinary conclusion in the premiss-sequent on the left but as a special modal premiss in the right-hand premiss-sequent.

In $\S 1$, the possibility was mentioned of regarding the schema $\succ_{\varphi} \square \varphi$ as encoding the rule of Necessitation. To construe the 'yields' relation as thus expressing the idea of 'yielding by the application of certain (formula-to-formula) rules', we should naturally expect this relation to be both reflexive and transitive,

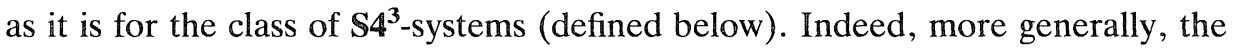
operation $C n$ defined by $C n(\Sigma)=\left\{\sigma \mid \vdash_{\Sigma} \sigma\right\}$ is, as the notation suggests, a consequence operation (in the sense of Tarski) for such systems. More generally still, for these systems, the (restriction to pairs of finite sets of the) relation $\Vdash$ defined by

$$
\Sigma \Vdash \Delta \Leftrightarrow \vdash_{\Sigma} \Delta, \text { is a }
$$

consequence relation in the sense of Scott. (See [12] for these notions.)

Intriguingly, we have found that although if attention is restricted to systems closed under the operational rules (offered in \$4) for ' $\square$ ', then closure under (Cross-cut) is all one needs to appeal to in order to get completeness results w.r.t. transitive structures, without such a restriction, a stronger structural rule than (Cross-cui) appears to be needed. Further, as has already been intimated, it does not appear to be possible, unless we so restrict our attention, to retain the same conception of a canonical structure for a system as was deployed in $\$ 2$. On the other hand, if we were to assume that $S$ was closed under the operational rules for ' $\square$ ', it would again turn out that no modification was required. We make these alternations - strengthening (Cross-cut) and reconstruing the relations $R_{S}$-because it seems desirable not to have to interrupt the 'purely structural' approach we have been taking to modal sequent logic.

First, them, the strengthened version of (Cross-cut), achieved by weakening its left-premiss: 
(4-cut)

$$
\frac{\Sigma \succ_{\Sigma} \sigma \quad \Gamma \succ_{\Sigma^{\prime}, \sigma} \Delta}{\Gamma \succ_{\Sigma, \Sigma^{\prime}} \Delta}
$$

Observe that this rule not only strengthens (Cross-cut) by a weakening of its left premiss (with a thinning on the left), but may at the same time be seen as a strengthening of (Undercut), again by a weakening of its left premiss (by a thinning 'below'). Any $\mathbf{K}^{3}$-system closed under (4-cut) - or equivalently, given the observation just made, any systems closed under $(\boldsymbol{R}),(\boldsymbol{M}),(\mathbb{T})$ and (4-cut) we will call a $\mathbf{K}^{3}$-system, and if it is also a $\mathrm{KT}^{\mathbf{3}}$-system, we will call it an $\mathbf{S 4 ^ { 3 }}$ system. As a familiarization exercise with the new rule, we suggest that the reader now verify that if the premiss-sequents of an application of (4-cut) hold in a structure $\langle W, R, V\rangle$ with $R$ transitive, then so does the conclusion-sequent.

Looking now in the direction of a completeness theorem in the same vein as Theorems 1 and 2, we introduce the new notion of canonical structure. For a consistent system $S$, the triple $\left\langle W_{S}, R_{S}, V_{S}\right\rangle$ will be its canonical structure in the new sense if $W_{S}$ and $V_{S}$ are exactly as in $\S 2$, while for $R_{S}$ we put:

$$
\langle\Gamma, \Sigma, \Delta\rangle R_{S}\left\langle\Gamma^{\prime}, \Sigma^{\prime} \Delta^{\prime}\right\rangle \text { iff } \Sigma \subseteq \Gamma^{\prime} \cap \Sigma^{\prime} .
$$

Note that, so defined, $R_{S}$ is transitive. (This is the case for any $\mathbb{K}^{3}$-system $S$. For $K 4^{3}$-systems closed under the operational rules for ' $\square$ ', there is no problem in showing that the relation $R_{S}$ as defined in $\S 2$ is transitive as well, as we shall show in $\S 4$, but the difficulty alluded to two paragraphs back was over the impossibility of showing this for arbitrary $\mathbb{K} 4^{3}$-systems tout court.) Since we have guaranteed the transitivity of $R_{S}$ by our new definition, it is clear that the job of exploiting the (4-cut) rule in the completeness argument will lie elsewhere (unlike the case of Theorem 2) and indeed we press it into service to show that an analogue of Lemma $\mathbb{B}$, vital for securing the crucial Lemma $C$, holds in the present setting. Let us call this:

Lemma $\mathbb{B}^{\prime}$. Where $S$ is any $\mathbf{K}^{3}$-system and $\langle\Gamma, \Sigma, \Delta\rangle$ is any maximal $S$-consistent triple, $\Sigma=\cap\left\{\Gamma^{\prime} \mid\right.$ for some $\Delta^{\prime},\left\langle\Gamma^{\prime}, \Sigma^{\prime}, \Delta^{\prime}\right\rangle$ is maximal $S$-consistent and $\left.\Sigma \subseteq \Gamma^{\prime} \cap \Sigma^{\prime}\right\}$

Proof. That $\Sigma$ is included in the intersection is again obvious. For the converse, suppose $\sigma \notin \Sigma$. Then, by maximality, $\Gamma \vdash_{\Sigma, \sigma} \Delta$. Now, if it were also the case that $\Sigma \vdash_{\Sigma} \sigma$, (4-cut) would give the conclusion that $\Gamma \vdash_{\Sigma} \Delta$, which we know not to be so; therefore $\Sigma H_{\Sigma} \sigma$. Thus $\langle\Sigma, \Sigma,\{\sigma\}\rangle$ is $S$-consistent and so extends to a maximal $S$-consistent triple $\left\langle\Gamma^{\prime}, \Sigma^{\prime}, \Delta^{\prime}\right\rangle$ with $\Sigma \subseteq \Gamma^{\prime} \cap \Sigma^{\prime}$ and $\sigma \notin \Gamma^{\prime}$ (since $\left.\sigma \in \Delta^{\prime}\right)$, as required.

Here we have helped ourselves to Lemma A, which holds unaltered, as does Lemma $C$ (with appeal to Lemma $B^{\prime}$ replacing the appeal to Lemma $B$ in the proof of Lemma $C$ in \$2). Without further ado, beyond observing that the 
reflexivity of the new $R_{S}$ is secured, as in Theorem 2, for $\mathbf{S 4}{ }^{3}$-systems by (Downward shift), we close with the analogous result for the present section.

Theorem 3. Any $\mathbf{K 4}^{3}$-system ( $\mathbf{S 4}^{3}$-system) is determined by the class of all its transitive structures (transitive reflexive structures).

Proof. As for Theorem 2, mutatis mutandis.

\section{§4. Some Operational Rules}

In this section, we give operational rules for the connectives, including ' $\square$ '. The rules for ' $\square$ ' do not vary from system to system, any more than the rules for, say, ' $\wedge$ ' do. Rather, they interact with the various structural rules of the previous sections to give rise to sequent formulations of the different modal logics.

To begin with the case of ' $\wedge$ ', there are many sets of rules closure under which secures completeness w.r.t. the usual truth-functional interpretation of this connective; for example, this is so for the following rules, here stated, as befits the three-place sequent formalism, with ' $\Sigma$ ' as an idle parameter:

$$
\frac{\Gamma, \varphi \wedge \psi \succ_{\Sigma} \Delta}{\overline{\Gamma, \varphi, \psi \succ_{{ }_{\Sigma}} \Delta}}
$$

The double line indicates that we are schematizing a rule for passing from the top sequent to the bottom sequent, and also a rule for passing from the bottom sequent to the top sequent.

As is well known, assuming $(\boldsymbol{R}),(\boldsymbol{M})$ and $(\mathbb{T})$, a further simplification is possible, replacing the above one-premiss rules by three zero-premiss (or 'unconditional') rules, namely:

$$
\varphi \wedge \psi>\varphi \quad \varphi \wedge \psi>\psi \quad \varphi, \psi>\varphi \wedge \psi
$$

(We do not need to insert the ' $\Sigma$ ' variable here, since we can 'thin' it in. ${ }^{9)}$ The interest, for our purposes, in the replacement here described is the guarantee it provides, for any system to which all their instantiations belong, that any structure for the system will, as far as ' $\wedge$ ' is concerned, be a model for the system: that is, the valuation of the structure will assign the value $T$ to a conjunction iff it assigns the value $T$ to each conjunct. A similar point holds for ' $\neg$ ', in view of the schemata: $\varphi, \neg \varphi>$ and $\succ \varphi, \neg \varphi$. (These zero-premiss rules replace the rules governing $\neg$ in, for example, [11], p.798.) Thus if the only connectives we had to deal with were ' $\wedge$ ' and ' $\neg$ ', we could say that any system closed under the appropriate rules had the desirable property that all its structures were 
amongst its models. A complete set of rules for ' $\square$ ', however, as we shall see below, do not secure this property. Before turning to those rules, we make the observation that any $\mathbf{K}^{\mathbf{3}}$-system closed under the rules for ' $\wedge$ ' will also be closed under the following two-way rule:

$$
\frac{\Gamma \succ_{\Sigma, \sigma \wedge \tau} \Delta}{\Gamma \succ_{\Sigma, \sigma, \tau} \Delta}
$$

(A demonstration of this fact appeals to (Undercut).) This can be thought of as a way of saying that necessity distributes over conjunction, without actually mentioning necessity.

This brings us to necessity itself, for which we have selected the following pair of rules:

$$
\frac{\Gamma, \square \sigma \succ_{\Sigma} \Delta}{\Gamma \succ_{\Sigma, \sigma} \Delta}
$$

The downward rule we shall call ' $(\square \downarrow)$ ), with ' $\square \uparrow$ )' for the upward direction. It is clear that both rules preserve the property of holding at a point in an arbitrary model - and a fortiori preserves the property of holding in a model no less than do the more familiar rules for ' $\wedge$ ' and ' $\neg$ ' already cited. Further, the rule $(\square \downarrow)$ can be replaced by a zero-premiss rule, the schema:

$(\square \downarrow)_{0}$

$>{ }_{\sigma} \square \sigma$

in the sense that closure under $(\square \downarrow)$ implies and is implied by closure under $(\square \downarrow)_{0}$ for any $\mathbb{K}^{3}$-system, in virtue of $(\mathbb{R})$, in the one case, and (T) (together with $(\mathbb{R})$ ) in the other. It is not, however, possible to replace ( $\square \uparrow$ ) with such a schema. In the light of what was said above about ' $\wedge$ ' and ' $\neg$ ', it is evident that if this were possible, then it would follow that every structure for any $\mathbb{K}^{3}$-system closed under the rules ( $\square$ ) was a model for that system. We shall see, with a simple example presented at the end of this section, that this not the case. In $\$ 5$, we explore a shift from three-place to four-place sequents which offers a remedy in this regard (Theorem 7 ).

We need some notation for systems closed under the operational rules for $\wedge, \neg$, and $\square$. $\mathbb{A} \mathbb{K}^{3}$-system so closed, we will call a $\mathbb{K}_{\square}^{3}$-system, a $\mathbb{K} \mathbb{T}^{3}$-system so closed, a $\mathbb{K}_{{ }^{\prime}}^{3}$-system, and so on. (Obviously, a more sensitive nomenclature would discriminate in the subscripting between the requirement of closure under the rules for ' $\neg$ ', under those for ' $\wedge$ ', and under those for ' $\square$ '.) If we have introduced a class of $X$-systems, for some label ' $X$ ', then we denote the smallest such system by ' $[X]$ '. Our three-place versions of the familiar modal logics $\mathbb{K} \mathbb{T}$ and $\mathbb{S} 4$ are thus denoted $\left[K_{\mathbb{T}}^{3}\right]$ and $\left[\mathbb{S}_{\square}^{3}\right]$, for example. However, the relationship between the modal principles used to define those familiar systems, and our own 'structural' approach may not be immediately obvious, so we elaborate on this a little here. 
For definiteness, we take the antecedently familiar approach to be that of two-place sequents $\Gamma>\Delta$ (see [8], for example); the normal modal logics in this framework, which analogy with our own usage might be called $\mathbf{K}^{2}$-systems, are those closed under the (non-structural!) rule:

(Normality)

$$
\frac{\varphi_{1}, \ldots, \varphi_{n}>\psi}{\square \varphi_{1}, \ldots, \square \varphi_{n}>-\square \psi} \quad n \geq 0
$$

To derive this rule in our framework, apply (Undercut) to its premiss-sequent and the $(\square \downarrow)_{0}$ sequent $\succ_{\psi} \square \psi$, giving

$$
>_{\varphi_{1} \ldots, \varphi_{n}} \square \psi
$$

from which the conclusion-sequent follows by $n$ applications of ( $\square \uparrow$ ). A sequent form of the familiar $\mathbf{T}$ principle is shown to belong to any $\mathrm{KT}_{\square}^{3}$-system thus:

$$
\begin{aligned}
\sigma & \succ \sigma & & (\boldsymbol{R}) \\
& \succ{ }_{\sigma} \sigma & & \text { (Downward shift) } \\
\square \sigma & \succ \sigma & & (\square \uparrow)
\end{aligned}
$$

Of course the second line here is the schema $\left(\mathrm{T}^{3}\right)$; we give the three-line proof for the sake of comparison with the following, which shows that the converse sequent belongs to any $\mathbb{K T}_{c_{\square}}^{3}$-system:

$$
\begin{aligned}
\square \sigma & \succ \square \sigma & & (\mathbb{R}) \\
& \succ{ }_{\sigma} \square \sigma & & (\square \downarrow) \\
\sigma & \succ \square \sigma & & \text { (Upward shift) }
\end{aligned}
$$

As our final illustration, we consider the 4 schema $\square \varphi \succ \square \square \varphi$. To show that an arbitrary sequent of this form belongs to any $4_{\square}^{3}$-system, we note that by the transitivity of the 'yields' relation (in \$3) for any such system, from the two $(\square \downarrow)_{0}$ sequents $>_{\varphi} \square \varphi$ and $>_{\square \varphi} \square \square \varphi$, we obtain $>_{\varphi} \square \square \varphi$, whence the desired result follows by ( $\square \uparrow$ ). In terms of our officially baptized rules, this appeal to the transitivity of 'yields' is a special case of the rule (Cross-cut) from $\S 3$. We do not need to appeal to the definitive (4-cut) rule which was described as stronger in the context of $\mathbb{K}^{3}$-systems and which we are about to see is not stronger in the context of $\mathbb{K}_{\square}^{\mathbf{3}}$-systems. To show that closure, for such systems, under (Cross-cut) implies closure under (4-cut), we use the fact, just demonstrated, that the sequents $\square \varphi>\square \square \varphi$ are derivable from (Cross-cut); from these in turn, we shall derive (4-cut). In virtue of $(M)$, it suffices to derive ( 4 -cut) in the special $\Sigma=\Sigma^{\prime}$ case - that is, to show that any (Cross-cut)-closed system containing $\Sigma>_{\Sigma} \sigma$ and $\Gamma>_{\Sigma, \sigma} \Delta$ also contains $\Gamma>_{\Sigma} \Delta$; taking $\Sigma=\left\{\sigma_{1}, \ldots, \sigma_{n}\right\}$ and writing $\square \Sigma$ for $\left\{\square \sigma_{1}, \ldots, \square \sigma_{n}\right\}$ : 
$\Sigma \succ_{\Sigma} \sigma$ (Given by hypothesis)

$\frac{\Sigma, \square \Sigma \succ \sigma((\square \uparrow) n \text { times }) \quad \Gamma \succ_{\Sigma, \sigma} \Delta}{\Gamma \succ_{\Sigma, \square \Sigma} \Delta}$

(Given by hypothesis)

(Undercut)

$\Gamma$, ㅁ $\Sigma \succ_{\Sigma} \Delta$

( $n$ applications of $(\square \uparrow)$

whence, since we have each sequent $\square \sigma_{i}>\square \square \sigma_{i}(1 \leq i \leq n)$, by $n$ applications of $(T)$ :

$$
\Gamma, \square \Sigma \succ_{\Sigma} \Delta
$$

and then a further $n$ applications of $(\square \downarrow)$ :

$$
\Gamma \succ_{\Sigma, \Sigma} \Delta, \text { i.e., } \Gamma \succ_{\Sigma} \Delta \text {. }
$$

So much for extracting more familiar looking modal principles out of our structural rules and the rules ( $\square$ ). Before passing to the topic of completeness, we indicate why the rules $(\square)$ commend themselves as appropriate. Their appropriateness on semantic grounds will already be evident. They do nothing more then directly reflect the intended interpretation of ' $\square$ ', ( $\square \downarrow)$ reflecting the sufficiency of the condition that $\varphi$ be true at all accessible points for the truth of $\square \varphi$ at a given point, and ( $\square \uparrow)$ reflecting the sufficiency of that condition. However, there is also a purely proof-theoretic side to the picture: these rules uniquely characterize ' $\square$ ' in the sense that for any singulary connectives ' $\square_{1}$ ' and ' $\square_{2}$ ' both governed by these rules, we have $\square_{1} \varphi \vdash \square_{2} \varphi$ and conversely. ${ }^{10)}$ For, by $(\mathbb{R})$, a system in the language of both these operators contains $\square_{1} \varphi>\square_{1} \varphi$, whence by $(\square \downarrow)$ for $\square_{1}$ : $\succ_{\varphi} \square_{1} \varphi$, and then, applying ( $\left.\square \uparrow\right)$ for $\square_{2}$, we get $\square_{2} \varphi>\square_{1} \varphi$. The derivation of the converse sequent of course uses ( $\left.\square \downarrow\right)$ for $\square_{2}$ and $(\square \uparrow)$ for $\square_{1}$. While the truth-functional connectives are thus uniquely characterized by the rules governing them in traditional (two-place) sequentlogical approaches (sequent-calculi, natural deduction, etc.), it can be shown that no such approach can uniquely characterize ' $\square$ ' for any but the Postcomplete normal modal logics (in which, in effect, this connective itself receives a truth-functional interpretation). This strongly suggests that the move from truth-functional to modal logic is not one best made simply by adding a new primitive connective with new rules governing it, but rather by extending one's conception of the objects to be manipulated by such rules. Hence the novel form of the sequents we have been working with.

We turn now to the topic of completeness. It is not difficult to convert Theorems 1-3 into results pertaining to models instead of structures in general, when closure under the operational rules presented in this section is assumed for the systems concerned - to show, that is, that any $\mathbf{K}_{\square}^{\mathbf{3}}$-system is determined by the class of all its models, any $\mathrm{KD}_{\square}^{3}$-system by the class of all its reflexive transitive models, and so on. Given those earlier theorems, it suffices to show that the canonical structures for the systems are indeed models. The latter 
involves showing that their valuations treat $\wedge, \neg$, and $\square$ compounds as required by $[\wedge],[\neg]$ and $[\square]$ in $\S 1$. The only case calling for some thought here is the last, which we settle in Lemma $D$, after noting that the common form of Lemmas $\mathrm{B}$ and $\mathrm{B}^{\prime}$ from $\S 2$ and $\S 3$, respectively, may be stated thus:

(*) For all $\langle\Gamma, \Sigma, \Delta\rangle \in W_{S}$,

$$
\Sigma=\cap\left\{\Gamma^{\prime} \mid\langle\Gamma, \Sigma, \Delta\rangle R_{S}\left\langle\Gamma^{\prime}, \Sigma^{\prime}, \Delta^{\prime}\right\rangle \text { for some } \Sigma^{\prime}, \Delta^{\prime}\right\}
$$

Lemma D. For any $\mathbf{K}_{\square}^{\mathbf{3}}$-system $S$, whether $\left\langle W_{S}, R_{S}, V_{S}\right\rangle$ is defined as in $\S 2$ or as in $\S 3$, for $\langle\Gamma, \Sigma, \Delta\rangle \in W_{S}, V_{S}(\square \varphi,\langle\Gamma, \Sigma, \Delta\rangle)=T$ iff for all $\left\langle\Gamma^{\prime}, \Sigma^{\prime}\right.$, $\left.\Delta^{\prime}\right\rangle \in W_{S}$ such that $\langle\Gamma, \Sigma, \Delta\rangle R_{S}\left\langle\Gamma^{\prime}, \Sigma^{\prime}, \Delta^{\prime}\right\rangle, V_{S}\left(\varphi,\left\langle\Gamma^{\prime}, \Sigma^{\prime}, \Delta^{\prime}\right\rangle\right)=T$.

Proof. 'Only if': Suppose $V_{S}(\square \varphi,\langle\Gamma, \Sigma, \Delta\rangle)=T$. Then $\square \varphi \in \Gamma$ by the definition of $V_{S}$, so $\varphi \in \Sigma$, by ( $\left.\square \uparrow\right)$. Therefore $\varphi \in \Gamma^{\prime}$ for all $\left\langle\Gamma^{\prime}, \Sigma^{\prime}, \Delta^{\prime}\right\rangle$ such that $\langle\Gamma, \Sigma, \Delta\rangle R_{S}\left\langle\Gamma^{\prime}, \Sigma^{\prime}, \Delta^{\prime}\right\rangle$, by the $\subseteq$-direction of $(*)$, whence $V_{S}\left(\varphi,\left\langle\Gamma^{\prime}\right.\right.$, $\left.\left.\Sigma^{\prime}, \Delta^{\prime}\right\rangle\right)=T$ for each such $\left\langle\Gamma^{\prime}, \Sigma^{\prime}, \Delta^{\prime}\right\rangle$, by the definition of $V_{S}$.

'If': Suppose $V_{S}\left(\varphi,\left\langle\Gamma^{\prime}, \Sigma^{\prime}, \Delta^{\prime}\right\rangle\right)=T$ for each $\left\langle\Gamma^{\prime}, \Sigma^{\prime}, \Delta^{\prime}\right\rangle$ such that $\langle\Gamma, \Sigma, \Delta\rangle$ $R_{S}\left\{\Gamma^{\prime}, \Sigma^{\prime}, \Delta^{\prime}\right\rangle$. Then (by Def. $\left.V_{S}\right) \varphi \in \Gamma^{\prime}$ for each such $\left\langle\Gamma^{\prime}, \Sigma^{\prime}, \Delta^{\prime}\right\rangle$, and so (by the $\supseteq$-direction of (*)), $\varphi \in \Sigma$. Therefore by $(\square \downarrow), \square \varphi \in \Sigma$, whence (by Def. $\left.V_{S}\right) V_{S}(\square \varphi,\langle\Gamma, \Sigma, \Delta\rangle)=T$.

In fact, as was mentioned in $\S 3$, it is not necessary to alter the definition of $R_{S}$ in $\$ 2$ in order to deal with $\mathbb{K} 4_{\square}^{3}$-systems, thanks to their closure under the operational rules, as indicated by the ' $\square$ ' subscript. The relation $R_{S}$ as defined in $\$ 2$ is already transitive for such $S$, and indeed coincides with the definiens for $R_{S}$ offered in $\$ 3$. For it is not hard to show that given any $\langle\Gamma, \Sigma, \Delta\rangle,\left\langle\Gamma^{\prime}, \Sigma^{\prime}\right.$, $\left.\Delta^{\prime}\right\rangle \in W_{S}$, for $S$ a $\mathbb{K}_{\square}^{3}$-system, if $\Sigma \subseteq \Gamma^{\prime}$ then $\Sigma \subseteq \Sigma^{\prime}$. (Supposing that $\Sigma \subseteq \Gamma^{\prime}$ and $\sigma \in \Sigma$, with a view to showing that $\sigma \in \Sigma$ ', begin by noting that $\square \sigma \in \Gamma$, and hence-exploiting the ' 4 ' property-that $\square \square \sigma \in \Gamma$; we leave the reader to complete the argument.)

We have not bothered to label the converted (structures-to-models) versions of Theorems 1,2, and 3 as a spearate Theorem. We turn to completeness results which fall out from the discussion for the specific systems $\left[\mathbf{K}_{\square}^{\mathbf{3}}\right],\left[\mathbf{K T}_{\square}^{3}\right]$, etc.

Theorem 4. The systems $\left[\mathbf{K}_{\square}^{3}\right],\left[\mathbf{K D}_{\square}^{3}\right],\left[\mathbf{K T}_{c \square}^{3}\right]\left[\mathbf{K T}_{\square}^{\mathbf{3}}\right],\left[\mathbf{K V e r}_{\square}^{3}\right],\left[\mathbf{K}_{\square}^{3}\right]$, and [S4 $\left.\mathbf{S}_{\square}^{3}\right]$ are determined by the classes of models $\langle W, R, V\rangle$ where, respectively, $R$ is arbitrary, $R$ is serial, $R$ is reflexive, $R$ is included in the identity relation, $R$ is the identity relation, $R$ is the empty relation, $R$ is transitive, and $R$ is both transitive and reflexive.

Proof. Soundness: Since the system $[X]$ is the least system satisfying the closure conditions on being an $X$-system (taking $X=\mathbf{K}_{\square}^{\mathbf{3}}, \mathbf{K T}_{\square}^{\mathbf{3}}$, etc.), an inductive proof is possible, showing that the rules formulating those conditions preserve the property of holding in a model of the class in question. We have already 
been through the details for the case of $\left[\mathbb{K}_{\square}^{3}\right]$ in showing (in $\left.\S 2\right)$ that $(\boldsymbol{R}),(\boldsymbol{M})$, $(T)$, and (Undercut) preserve the property of holding in any structure, and a fortiori in any model, and noting (in the present section) that all our operational rules preserve the property of holding in any model. We have also noted in $\$ 2$ and $\S 3$ that the rules defining $\mathrm{KT}_{\square}^{3}$-systems and $\mathbf{K}_{\square}{ }^{3}$-systems enjoy this feature w.r.t. those models which are, respectively, reflexive and transitive. The remaining cases are similar.

Completeness: This is established by the remarks leading up to the proof of Lemma $\mathrm{D}$, which show that the canonical structures for these systems are in fact models for the systems. For the cases of $\left[\mathbf{K T}_{c \square}^{3}\right]$ and $\left[\mathbf{K T}_{\square}^{\mathbf{3}}\right]$, we exploit closure under the rules for $\neg$, recalling the comments following Theorem 2 .

The completeness results collected in Theorem 4 were derived from the observation that the canonical structures for the systems concerned were models for those systems. This by no means implies that every structure for any such system is a model, and in fact this is false. The following is a structure for [ $\left[\mathbb{K}_{a}^{\mathbf{3}}\right]$ (indeed, for [ $\left[\mathrm{KVer}^{3}\right]$ ) which falls short of being a model. For some object $w$, let $W=\{w\}, R_{1}=\{\langle w, w\rangle\}$, and $V$ be the unique valuation on $W$ such that:

$$
\begin{aligned}
& V\left(p_{i}, w\right)=F \text { for all propositional variables } p_{i} \\
& V(\varphi \wedge \psi, w)=T \text { iff } V(\varphi, w)=V(\psi, w)=T \\
& V(\neg \varphi, w)=T \text { iff } V(\varphi, w)=F \\
& V(\square \varphi, w)=T \text { for all formulae } \varphi .
\end{aligned}
$$

Clearly the structure $\left\langle W, R_{1}, V\right\rangle$ is not a model, since, for example, $V\left(\square p_{1}\right.$, $w)=T$ while for some $y \in W$ (namely, $w$ itself) we have $w R_{1} y$ and $V\left(p_{1}, y\right)=F$. It remains to show that $\left\langle W, R_{1}, V\right\rangle$ is a structure for $\left[\mathbb{K}_{\square}^{3}\right]$. To that end, consider the different structure $\left\langle W, R_{0}, V\right\rangle$ where $R_{0}$ is the empty relation on $W$. Note that this structure is a model: so every sequent in $\left[\mathbb{K}_{\square}^{3}\right]$ holds in it. The result we seek - that every such sequent holds in the original struture - will follow if we can show that any sequent holding in $\left\langle W, R_{0}, V\right\rangle$ holds in $\left\langle W, R_{1}, V\right\rangle$ : but this is immediate.

To the extent, then, that one sees it as a desideratum that one's logical framework should provide notions of structure and model according to which every structure for a system closed under a complete set of operational rules must be a model for that system, one will be dissatisfied with the framework we have so far provided, the various completeness theorems notwithstanding. As we shall see, the three-place sequent framework may be viewed as something of a half-way house in this regard, between the more conventional two-place framework and something richer still. 


\section{§5. Four-Place Sequents}

The development thus far has perhaps conveyed the respects in which our treatment of modal logic using three-place sequents improves on more traditional approaches, by distinguishing special modal ( $\Sigma$-position $)$ premisses from 'ordinary' ( $\Gamma$-position) premisses. A certain refinement of that treatment will now be suggested, invoking a similar distinction amongst conclusions, which retains its benefits (unique characterization of ' $\square$ ', smooth mesh between semantics and proof-theory, etc.) while eliminating such anomalies as remain: for example, the existence of structures for logics which are closed under the operational rules, which structures fail to be models. Further, as will hardly have escaped the reader's notice, our sampling of the range of normal modal logics has been very selective, and conspicuously absent from that selection was any account of the $\mathbf{B}$ ('Brouwersche') principle, $\varphi>\square \neg \square \neg \varphi$, (or, for short: $\varphi>\square \backslash \varphi$ ). Likewise for the 5 schema: $\langle\varphi>\square \diamond \varphi$ Thus no route was provided to that best-loved of all modal logics, S5. While there would be no difficulty in adding such principles as they stand, we are naturally more interested in achieving their effect by the use of structural rules, and the four-place sequents with which we shall now be working provide a framework in which the desired rules can be formulated.

From this point on, a sequent will be an object $\Gamma>{ }_{\Sigma}^{\Theta} \Delta$, and our systems will be certain collections of these; their labels will bear a superscripted ' 4 ' to replace the ' 3 ' of the sequent systems in the three-place framework hitherto dominant. In connexion with such a system, the metalinguistic notation $\Gamma \vdash \stackrel{\Theta}{\Theta} \Delta$ is to be understood analogously to its three-place namesake. Semantic concepts defined in terms of the notion of a structure are to be understood exactly as in $\S 1$, though of course, we need a new definition of what it is for a sequent to hold at a point in such a structure, and in fact say that $\Gamma>-\underset{\Sigma}{\Theta} \Delta$ holds at $x$ in the structure $\langle W, R, V\rangle$ iff:

If for all $\varphi \in \Gamma, V(\varphi, x)=T$ and for all $\sigma \in \Sigma V(\sigma, y)=T$ for each $y \in W$ such that $x R y$, then either there exists $\psi \in \Delta$ such that $V(\psi, x)=T$ or for some $\theta \in \Theta, V(\theta, y)=T$ for each $y \in W$ such that $x R y$,

In more colloquial terms, the sequent holds just in case if all of $\Gamma$ are true and all of $\Sigma$ are necessary, then either some of $\Delta$ are true or else some of $\Theta$ are necessary.

A collection of (four-place) sequents is a $\mathbb{K}^{4}$-system iff it is closed under the following rules:

$(\boldsymbol{R})$

$(M)$

$$
\begin{aligned}
& \varphi>\varphi \\
& \frac{\Gamma>{ }_{\Sigma}^{\Theta} \Delta}{\Gamma, \Gamma^{\prime}>{\underset{\Sigma, \Sigma^{\prime}}{\Theta} \Delta, \Theta^{\prime}}^{\prime} \Delta, \Delta^{\prime}}
\end{aligned}
$$

$($ Vertical $\boldsymbol{R}) \quad \succ_{\sigma}^{\sigma}$

(Undercut) $\frac{\Sigma \succ \sigma \quad \Gamma \succ_{\Sigma^{\prime}, \sigma}^{\Theta} \Delta}{\Gamma \succ{ }_{\Sigma, \Sigma^{\prime}}^{\Theta} \Delta}$ 


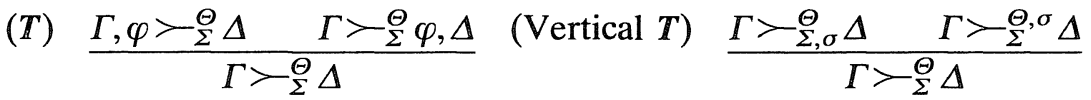

As to alternative definitions of this same class of systems, we confine ourselves to the observation that closure under the above rules (in particular, (Undercut) and (Vertical $\boldsymbol{R}$ )) implies closure under this rule:

(Switch)

$$
\frac{\Sigma \succ \sigma}{\succ_{\Sigma}^{\sigma}}
$$

and that, conversely, (Switch) delivers (Vertical $\mathbb{R}$ ) from $(\boldsymbol{R})$ and (Undercut) from (Vertical $T$ ). We remark that (Switch) can be thought of as a ' $\square$ '-free version of the rule (Normality) in the preceding section.

The following result is proved by a straightforward modification of the canonical structure methods of $\$ 1$, except that now such structures should consist of quadruples $\langle\Gamma, \Sigma, \Theta, \Delta\rangle$ where the sets involved are maximally such that $\Gamma H_{\Sigma}^{\Theta} \Delta$; the same definition of $R_{S}$ as in $\$ 2$ will do. For the analogue of Lemma $A$ in $\$ 2$, the new rules (Vertical $\mathbb{R}$ ) and (Vertical $\mathbb{T}$ ) need to be exploited (as well as $(\mathbb{M})$ : these have the effect that the $\Sigma$ and $\Theta$ of such maximal quadruples are complements).

Theorem 5. Any $\mathbb{K}^{4}$-system is determined by the class of all its structures.

This is the analogue of Theorem 1 in $\$ 2$. Rather than repeating the analogues of Theorems 2 and 3, we pass straight to a case not treated before, and define a $\mathrm{KB}^{4}$-system to be one closed under the rule:

$$
\frac{\Sigma \succ^{\Delta} \sigma \quad \Gamma>\succ_{\Sigma, \sigma}^{\Theta} \Delta}{\Gamma \succ{ }_{\Sigma}^{\Theta} \Delta}
$$

To get a feel for this rule, we recommend that the reader verify that whenever its premiss-sequents hold in a structure $\langle W, R, V\rangle$ in which $R$ is symmetric, so does the conclusion-sequent. This, together with the following Theorem, gives the result that $\left[\mathbf{K B}_{\square}^{4}\right]$ is determined by the class of all symmetric models, though the subscripting notation here has to be explained anew for the 4-place framework (see below).

Theorem 6. Any $\mathbb{K B}^{4}$-system is determined by the class of all its symmetric structures.

Proof. It will be enough to remark that we may define a symmetric relation $R_{s}$ which for the systems in question gives the appropriate analogues of Lemmas $\mathrm{B}$ and $\mathrm{C}$, by 


$$
\langle\Gamma, \Sigma, \Theta, \Delta\rangle R_{S}\left\langle\Gamma^{\prime} \Sigma^{\prime} \Theta^{\prime}, \Delta^{\prime}\right\rangle \text { iff } \Sigma \subseteq \Gamma^{\prime} \text { and } \Sigma^{\prime} \subseteq \Gamma
$$

As for the [ ]-notation in the present framework, we take over the unconditional rules for $\wedge$ and $\neg$ from $\S 4$, and understand by rules for $\square$, those stated as ( $\square$ ) in that section but with an additional variable for sets of formulae sitting in the upper ' $\Theta$ '-position (remaining unchanged from premiss-sequent to conclusionsequent). If we take over the label (4-Cut) from our discussion in $\S 3$ with a similar understanding (fixed $\Theta$-variable) and define a $\mathbf{K} \mathbf{4}^{\mathbf{4}}$-system to be a $\mathbf{K}^{\mathbf{4}}$ system closed under (Undercut) so understood, then for our version of S5, namely, $\left[\mathrm{KTB}^{4}\right]$, we have the result that this system is determined by the class of all models whose accessibility relation is an equivalence relation, as one would expect. (We omit details). However, the greatest theoretical significance of the use of the rules ( $\square$ ) in the four-place framework is not so much in the structural tractability of these new modal logics, as in the fact that now not only can $(\square \downarrow)$ be given an equivalent unconditional formulation (as $(\square \downarrow)_{0}$ ), but so can $(\square \uparrow)$. We call this $(\square \uparrow)_{0}: \square \varphi \succ^{\varphi}$. To obtain $(\square \uparrow)_{0}$ from $(\square \uparrow)$, apply the latter rule to the (Vertical $\mathbb{R}$ ) instance $\succ_{\varphi}^{\varphi}$. From the converse direction, we must start with $(\square \uparrow)_{0}$ and a premiss-sequent $\Gamma \succ_{\Sigma, \sigma}^{\Theta} \Delta$ of $(\square \uparrow)$, to derive the conclusion-sequent: $\Gamma, \square \sigma>{ }_{\Sigma}^{\Theta} \Delta$. The appropriate instance of $(\square \uparrow)_{0}$ with which to begin is $\square \sigma \succ^{\sigma}$, whence the desired conclusion follows by $(\boldsymbol{M})$ and (Vertical $T$ ). We close with a Corollary to the availability of these unconditional or zero-premiss equivalents to the rules ( $\square)$ :

\section{Theorem 7. Any structure for a $\mathbf{K}_{\square}^{4}$-system is a model.}

Notes 1) We thank T.E. Karmo for a correction incorporated in that revision.

2) For example, we pay no attention to questions of Cut-elimination: our interest in the various rules we present is in their tightness of fit with semantic notions rather than with such more traditional preoccupations of proof-theorists.

3) On the other hand, many features on which Belnap lays emphasis, such as the 'structure connectives' of [2], do not appear here.

4) That is, rules whose schematic statement requires no mention of any connectives. This usage (and the opposition with 'operational' rules) derives from Gentzen's work, and is to be sharply distinguished from the use of 'structural rule' originating in [9] to mean: rule such that any substitution-instance of an application of the rule is in turn an application of the rule.

5) The use of ' $\sigma$ ', as opposed to ' $\varphi$ '. ' $\psi$ ', etc., here and elsewhere has no official significance: we use ' $\sigma$ ', (and below ' $\tau$ '), for suggestive purposes only, generally when the formula in question plays a crucial role at some stage in the ' $\Sigma$ ' position of some sequent in the inference figure exhibited. Differences in the use of the set-of-formulae variables ' $\Gamma$ ', ' $\Delta$ ', ' $\Sigma$ ' (and, in $\$ 5, ~ ' \Theta ')$ are likewise themselves purely suggestive.

6) In the case of a zero-(sequent-)premiss rule such as $(\boldsymbol{R})$, this means simply that every instance of the schema $(\boldsymbol{R})$ holds in every structure. Similarly, below, when we speak of a system as closed under various sequent-to-sequent rules, we mean in the zero-premiss case that every instance of the schema in question belongs to the system.

7) We use here the nomenclature of [3], [13]. 
8) Several such anomalies for the two-place sequent framework (in which a semantic completeness result for a modal system requires the presence of certain boolean connectives) are presented in [7].

9) As in the case $(\boldsymbol{R})$ in $\$ 2$.

10) Attention was drawn to this notion by Belnap, with a credit to $\mathrm{H}$. Hiż, in [1]: sec [5], [6] for more recent signs of interest in the topic.

\section{References}

[1] Belnap, N.D., Tonk, Plonk, and Plink, Analysis, 22 (1962), 130-134.

[2] Display Logic Journal of Philosophical Logic, 11 (1982), 375-417.

[3] Chellas, B., Modal Logic: An Introduction. Cambridge University Press, 1980.

[4] Došen, K., Sequent-Systems for Modal Logic, Journal of Symbolic Logic, 50 (1985). 149-168.

[5] Došen, K., and P. Schroeder-Heister, Conservativeness and Uniqueness, Theoria, $5 \mathbb{1}$ (1985), $159-173$.

[6] Humberstone, I.L., Unique Characterization of Connectives (Abstract), Journal of Symbolic Logic, 49 (1984), 1426-1427.

[7] $\longrightarrow$ Expressive Power and Semantic Completeness: Boolean Connectives in Modal Logic, Studia Logica, 49 (1990), 197-214.

[8] Kuhn, S., Many-Sorted Modal Logics, Vol. I, Filosofiska Studier (29), Uppsala 1977.

[9] Los. J., and R. Suszko, Remarks on Sentential Logics. Indagationes Math.. 61 (1958), 177-183.

[10] Sato, M., A Study of Kripke-type Models for Some Modal Logics by Gentzen's Sequential Method, Publ. RIMS, Kyoto Univ., 13 (1977), 381-466.

[11] Scott, D.S., On Engendering an Illusion of Understanding, Journal of Philosophy, 68 (1971), 787-807.

[12] Completeness and Axiomatizability in Many-Valued Logic, in Procs. of the Tarski Symposium, ed. L. Henkin et al., American Math. Society (Providence, Rhode Island), 1974.

[13] Segerberg, K., An Essay in Classical Modal Logic, Filosofiska Studier (13), Uppsala 1971. 